\title{
Structure of nanosized materials by high-energy X-ray diffraction: study of titanate nanotubes
}

\author{
Milen Gateshki ${ }^{\mathrm{I}, 1}$, Qing Chen ${ }^{\mathrm{II}}$, Lian-Mao Peng ${ }^{\mathrm{IIII}}$, Peter Chupas ${ }^{\mathrm{III}}$ and Valeri Petkov ${ }^{*, \mathrm{I}}$ \\ I Department of Physics, Central Michigan University, Mt. Pleasant, Michigan 48859, USA \\ II Key Laboratory on the Physics and Chemistry of Nanodevices and Department of Electronics, Peking University, Beijing 100871, PR China \\ ${ }^{\text {III }}$ Advanced Photon Source, Argonne National Laboratory, Argonne, Illinois 60439, USA
}

Received May 16, 2007; accepted August 17, 2007

Synchrotron X-ray diffraction /

High energy X-ray diffraction / Nanotubes / Titanate

Abstract. High-energy X-ray diffraction and atomic Pair Distribution Function analysis are employed to determine the atomic-scale structure of titanate nanotubes. It is found that the nanotube walls are built of layers of $\mathrm{Ti}-\mathrm{O}_{6}$ octahedra simular to those observed in crystalline layered titanates. In the nanotubes, however, the layers are bent and not stacked in perfect registry as in the crystal.

\section{Introduction}

Titanate $\left(\mathrm{TiO}_{2}\right.$-type) nanotubes were first synthesized by alkaline hydrothermal reaction in 1998 [1, 2] and extensively studied since then [3-15] due to their useful properties. In particular, similarly to bulk crystalline $\mathrm{TiO}_{2}$ the nanotubes are a wide band gap semiconductor, a property finding application in photocatalysis. In addition, the nanotubes have been found to accommodate easily various atomic and ionic species. As such titanate nanotubes have a very good potential for applications ranging from lithium batteries, hydrogen storage to solar-cells [3, 4]. It is well known that properties of materials are, to a great extent, determined by their atomic-scale structure and that is why its knowledge is highly needed. Several transmission electron microscopy (TEM), electron and X-ray diffraction (XRD) experiments on titanate nanotubes have been conducted so far and a few qualitative structure models proposed [5-11]. None of the models, however, has been rigorously tested or refined against diffraction data. The reason is that traditional techniques for atomic-scale structure determination and refinement are very difficult, if not impossible, to apply to materials of nanosize dimensions or length of structural coherence. Apparently the diffraction patterns of such materials are so smeared that the traditional techniques relying mostly on sharp Bragg peaks turn inapplicable. Recently it was demonstrated that a

\footnotetext{
* Correspondence author (e-mail: petkov@phy.cmich.edu)

1 Present address: Bragg Institute, ANSTO, Menai, NSW 2234, Australia
}

non-traditional approach involving high-energy XRD and atomic Pair Distribution Function (PDF) analysis can yield the atomic-scale structure of nanophase materials [16-19] in detail. Here we apply this approach to titanate nanotubes.

\section{Experimental}

\section{Sample preparation}

Titanate nanotubes were prepared as follows: $\mathrm{TiO}_{2}$ particles (pure anatase or anatase and rutile) with size ranging from $10 \mathrm{~nm}$ to more than $200 \mathrm{~nm}$ were added to a $10-\mathrm{M}$ $\mathrm{NaOH}$ aqueous solution. The mixture was sealed in a Teflon container and statically heated in a furnace at $130{ }^{\circ} \mathrm{C}$ for $72 \mathrm{~h}$. The precipitate was filtered and washed at room temperature with different solvents, including water, ethanol, and acetone. As a result, a large quantity of nanotubes with a yield $>90 \%$ was obtained. As TEM images show (see Fig. 1) the nanotubes are multiwall with inner and outer diameters of approx. $6 \mathrm{~nm}$ and $9 \mathrm{~nm}$ (Fig. 1), respectively. More details of the preparation procedure can be found in [4-7].

\section{X-ray diffraction experiments}

Titanate nanotubes were measured on an X-ray diffractometer (PANalytical X'Pert) using $\mathrm{Cu} K_{\alpha}$ radiation and a

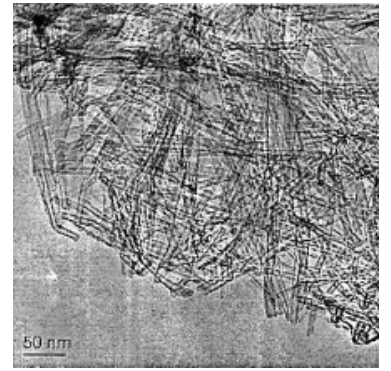

(a)

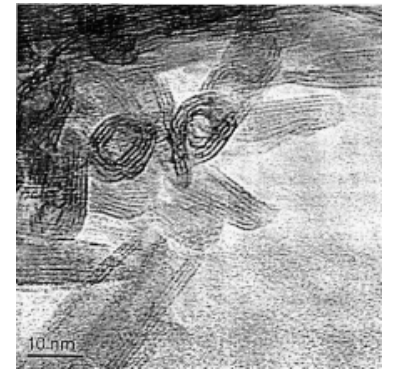

(b)
Fig. 1. TEM images of titanate nanotubes. The as prepared material is a pile of randomly oriented nanotubes (a). Most nanotubes are multiwall, several tens of nanometers long and approximately $9 \mathrm{~nm}$ in diameter (b). 


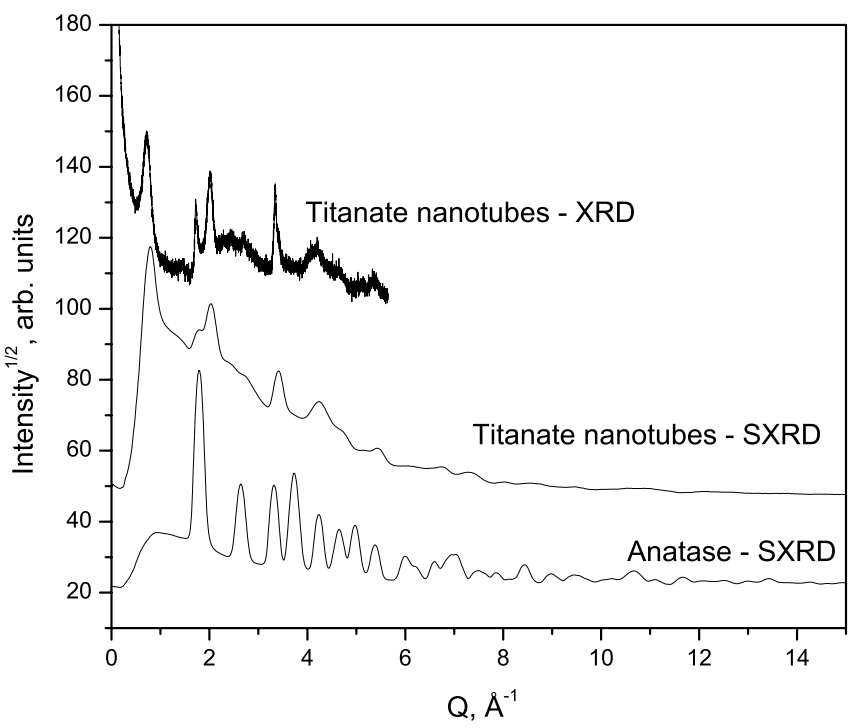

Fig. 2. Diffraction patterns of titanate nanotubes collected with $\mathrm{Cu} K_{\alpha}$ and synchrotron radiation (SXRD) X-rays. The SXRD pattern of polycrystalline bulk anatase material is also shown. Note the resolution of in house and SXRD patterns is different because of the different detectors used: point and large area, respectively. The patterns are shifted vertically for clarity.

point (scintillation) detector. The diffraction pattern, covering a wide range of diffraction angles $2 \theta$ (2-90 deg.) is shown in Fig. 2. It exhibits only a few sharp features and, as already observed in previous studies [3], does not provide a firm basis for structure search and refinement. Besides, it covers quite a limited range of reciprocal space vectors $Q=4 \pi(\sin \theta) / \lambda$, where $\theta$ is half the angle between the incoming and outgoing $\mathrm{X}$-rays, and $\lambda$ is the wavelength of the radiation used $\left(\lambda=1.54 \AA\right.$ for $\left.\mathrm{Cu} K_{\alpha}\right)$. A diffraction pattern of titanate nanotubes (see Fig. 2) obtained using synchrotron radiation $\mathrm{X}$-rays with energy of $90.48 \mathrm{keV}(\lambda=0.1372 \AA)$ covers a much wider region of reciprocal space vectors (see Fig. 2 and 3 ). The pattern was obtained at the beamline 11-ID-B at the Advanced Photon Source, Argonne National Laboratory. Besides, the higher flux of synchrotron radiation X-rays and the usage of a large area (General Electric) detector allowed to measure the rather diffuse XRD pattern of titanate nanotubes with a very good statistical accuracy. Both an extended range of $Q$ vectors and a very good statistical accuracy are a prerequisite to the atomic PDF data analysis [20] adopted here. Polycrystalline anatase (from Sigma; average crystallite size of $\sim 100 \mathrm{~nm}$ ) was also measured using synchrotron radiation X-rays (see Fig. 2) and used as a reference material.

\section{Results}

As can be seen in Fig. 2, the diffraction pattern of anatase standard exhibits well-defined Bragg peaks, as might be expected for a bulk crystal. Such a pattern lends itself to traditional crystallographic analysis. It has already revealed that anatase is a 3D network of both edge and corner sharing $\mathrm{Ti}-\mathrm{O}_{6}$ octahedra that may be described in terms of a tetragonal lattice (S.G. I4 $/$ amd) with a 12 atom unit cell of parameters $a=3.7867 \AA$ and $b=9.5149 \AA$ [21]. The diffraction pattern of titanate nanotubes, however, is too much diffuse to be analyzed in the traditional crystallographic way. That is why it was considered in terms of the corresponding total reduced structure factor $F(Q)=Q[S(Q)-1]$ and its Fourier transform - the atomic PDF $G(r)=4 \pi r\left[\varrho(r)-\varrho_{0}\right]$. Here $\varrho(r)$ and $\varrho_{0}$ are the local and average atomic number densities, respectively, and $r$ is the radial distance. The structure factor $S(Q)$ is related to the coherent part, $I^{\mathrm{coh}}(Q)$, of the diffraction data as follows:

$$
S(Q)=1+\left[I^{\mathrm{coh}}(Q)-\sum c_{i}\left|f_{i}(Q)\right|^{2}\right] /\left|\sum c_{i} f_{i}(Q)\right|^{2} .
$$

$c_{i}$ and $f_{i}$ are the atomic concentration and X-ray scattering factor, respectively, for the atomic species of type $i$ $[20,22]$. The reduced structure factor for titanate nanotubes extracted from the synchrotron radiation diffraction data is shown in Fig. 3a. The in-house obtained XRD data were not appropriate to be considered in terms of $F(Q) / G(r)$ because of their rather low $Q$ limit. The structure factor of bulk crystalline anatase is shown in Fig. 3a as well. The corresponding atomic PDFs $G(r)$ are shown in Fig. 3b. The processing of synchrotron XRD data and derivation of $F(Q) / G(r)$ was done with the help of the program RAD [23].

A comparison between the data sets of Fig. 2 and 3 exemplifies the different way the same diffraction features appear, and hence, are accounted for in structure studies relying on low- and high-energy XRD. XRD patterns obtained using low-energy $\mathrm{X}$-rays (e.g. $\mathrm{Cu} K_{\alpha}$ radiation) may cover only a limited range of wave vectors and, hence, are sensitive mostly to longer-range atomic ordering in materials. High-energy (e.g. synchrotron) XRD may reach much higher $Q$ vectors rendering the corresponding $F(Q) / G(r)$ a quantity that is very well suited to study materials of

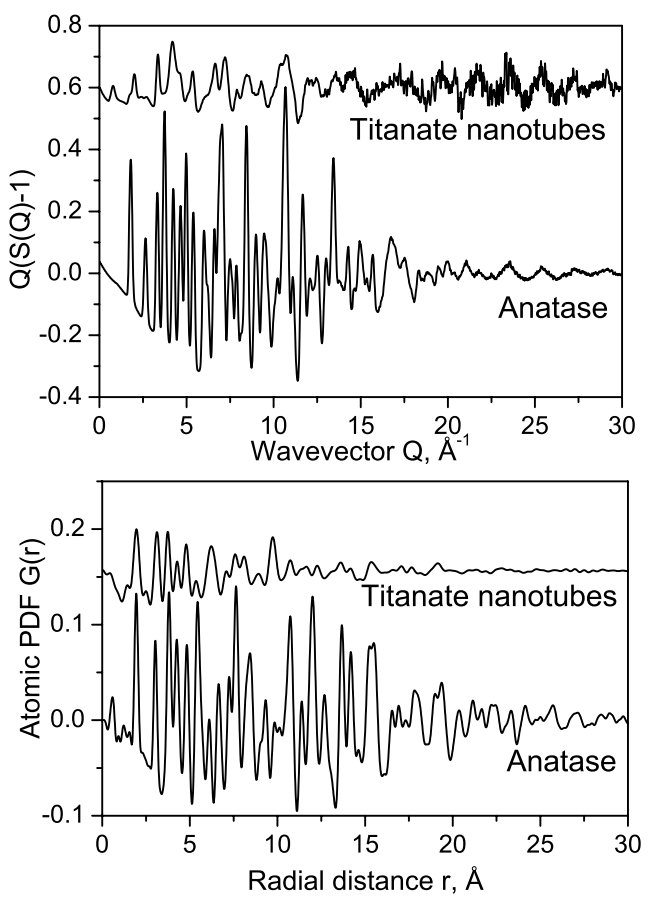

Fig. 3. Reduced structure factors (a) and the corresponding atomic PDFs (b) for titanate nanotubes and bulk crystalline anatase derived from the synchrotron XRD patterns in Fig. 2. 
limited, including nanoscale, length of structural coherence. That titanate nanotubes is such a material is clearly seen in Fig. 3b showing its PDF decaying to zero already at approximately $2 \mathrm{~nm}$. In contrast, the PDF for bulk anatase persists to much longer interatomic distances, as it should be with materials exhibiting a long-range (i.e. crystalline-type) atomic ordering. Regardless of its rapid decay, however, the PDF of the nanotubes still shows many well defined features allowing a structure search and refinement. For example, its first peak is positioned at about $1.9 \AA$ which is the $\mathrm{Ti}-\mathrm{O}$ distance in $\mathrm{Ti}-\mathrm{O}_{6}$ octahedra. A similar peak is present in the experimental PDF for anatase as well. This observation showed that, like anatase, the nanotube walls are built of well-defined $\mathrm{Ti}-\mathrm{O}_{6}$ octahedra. The experimental PDFs of titanate nanotubes and anatase, however, disagree substantially at longer interatomic distances indicating that the way $\mathrm{Ti}-\mathrm{O}_{6}$ octahedra are arranged in space is substantially different in the two materials, contrary to the suggestions of Kasuga et al. [1]. This result may not come as a surprise. Other oxide nanotubes like $\mathrm{V}_{2} \mathrm{O}_{5}$ have been found not to share the structure type of the corresponding bulk crystal either [24].

\section{Discussion}

Previous studies [3, 4] suggested that titanate nanotubes obtained through a hydrothermal reaction possess a layered and not a network-type structure as their crystalline counterparts do. Two somewhat different structure models have been proposed: one based on the lepidocrocite, $\mathrm{H}_{x} \mathrm{Ti}_{2-x / 4} \square_{x / 4} \mathrm{O}_{4}$, type structure (here $\square$ indicates a vacancy) [9] and the other - on the trititanate, $\mathrm{H}_{2} \mathrm{Ti}_{3} \mathrm{O}_{7}$, type structure [4]. Fragments from the two structure types are shown in Fig. 4.

As can be seen in Fig. 4 both materials are built of layers of $\mathrm{Ti}-\mathrm{O}_{6}$ octahedra. Layers in trititanate, $\mathrm{H}_{2} \mathrm{Ti}_{3} \mathrm{O}_{7}$, are composed of fragments of three edge-sharing octahedra. The fragments join at their corners in a step-wise manner (see Fig. 4a) rendering the layers corrugated. Layers in lepidocrocite, $\mathrm{H}_{x} \mathrm{Ti}_{2-x / 4} \square_{x / 4} \mathrm{O}_{4}$, too are made of edge-sharing $\mathrm{Ti}-\mathrm{O}_{6}$ octahedra but are pretty much flat (see Fig. 4c). It may be noted that these structure types are the end members of a series of layered titanates with a general formula $\mathrm{H}_{2} \mathrm{Ti}_{n} \mathrm{O}_{2 n+1}$, where $\mathrm{n}$ is the number of $\mathrm{Ti}$ atoms involved in the layer fragments. Another member of this series, $\mathrm{H}_{2} \mathrm{Ti}_{4} \mathrm{O}_{9}$, has also been considered as a model for the structure of titanate nanotubes [10]. The two distinct type models featuring corrugated and flat layers, respectively, were tested by computing the corresponding atomic PDFs and comparing them to the experimental PDF for titanate nanotubes. The calculations were done with the help of the program PDFFIT [25]. The structure model parameters including lattice constants, atomic positions and isotropic thermal factors were adjusted to achieve the best possible agreement between the model and experimental PDF data while keeping the inherent topology and symmetry of the models intact. The limited length of structural coherence in the nanotubes, including the presence of layer-layer stacking (turbostratic) disorder, was taken into account as discussed in [19]. A model PDF

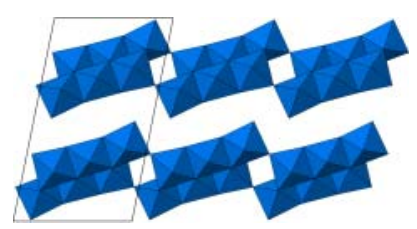

(a)

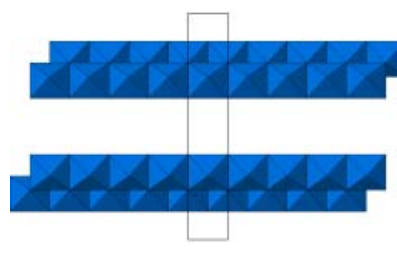

(C)

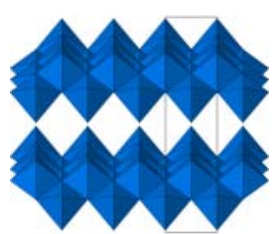

(b)

Fig. 4. Fragments from the crystal structures of $\mathrm{H}_{2} \mathrm{Ti}_{3} \mathrm{O}_{7}(\mathbf{a}, \mathbf{b})$ and $\mathrm{H}_{x} \mathrm{Ti}_{2-x / 4} \square_{x / 4} \mathrm{O}_{4}$ (c, d) layered titanates. The corresponding unit cells are outlined with thin solid lines.

based on the network-type anatase structure was computed as well. Results are presented in Fig. 5.

As can be seen in Fig. 5 a model based on the anatase structure does fail to reproduce the experimental PDF data. Models based on the rutile and brookite modifications of $\mathrm{TiO}_{2}$, that too are $3 \mathrm{D}$ networks of $\mathrm{Ti}-\mathrm{O}_{6}$ octahdera, did not perform any better. On the other hand, models featuring layers of octahedra reproduce the experimental data quite well. From the two distinct layer-type models the one based on fragmented/corrugated layers (i.e. $\mathrm{H}_{2} \mathrm{Ti}_{3} \mathrm{O}_{7}$-type) was found to perform somewhat better, especially at shorter interatomic distances. There are three crystallographically different $\mathrm{Ti}$ atoms and correspondingly three types of octahedra with different connectivity in $\mathrm{H}_{2} \mathrm{Ti}_{3} \mathrm{O}_{7}$. As a result, on average, each octahedron is con-

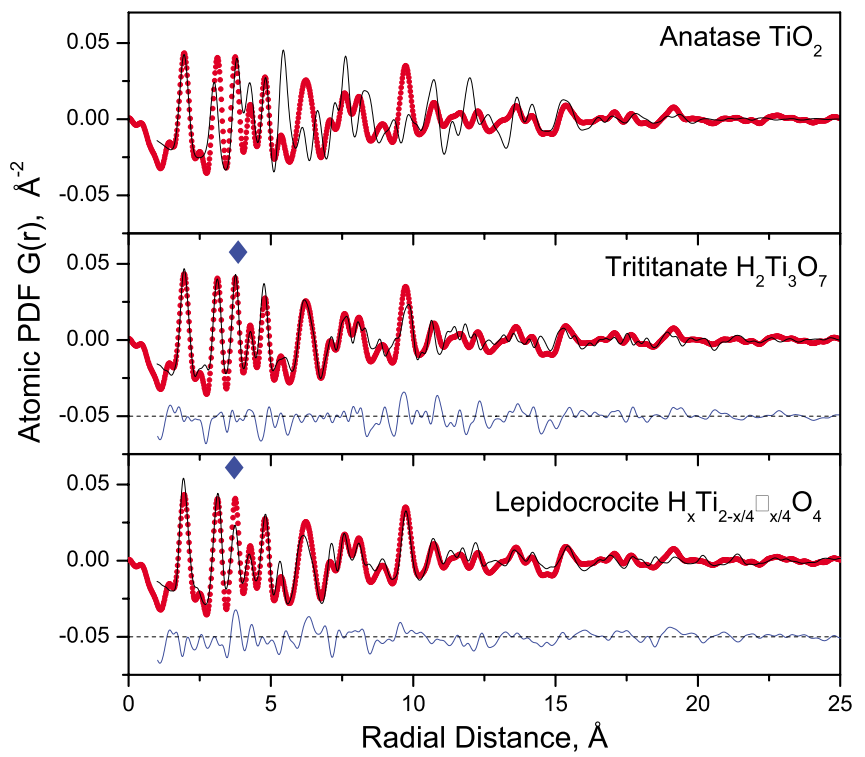

Fig. 5. Comparison between the experimental PDF for titania nanotubes (symbols in red) and model PDFs (black lines) based on the structures of anatase, trititanate and lepidocrocite crystals. Difference between the model and experimental data is given at the bottom (in blue). The PDF peak reflecting the correlations between $\mathrm{Ti}$ atoms from adjacent, corner-sharing octahedra is marked with a blue diamond. 


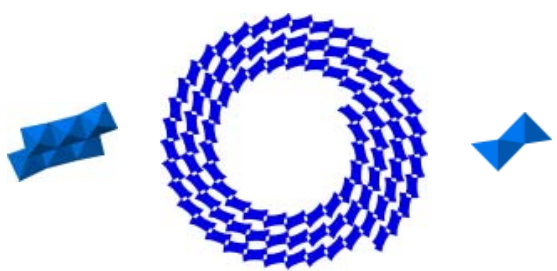

(a)

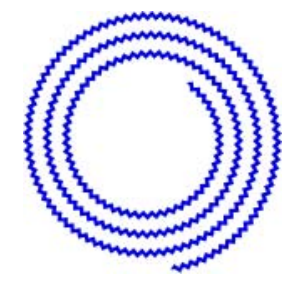

(b)

Fig. 6. Cross sections of the titanate nanotube models based on $\mathrm{H}_{2} \mathrm{Ti}_{3} \mathrm{O}_{7} /$ corrugated (a) and lepidocrocite $\mathrm{H}_{x} \mathrm{Ti}_{2-x / 4} \square_{x / 4} \mathrm{O}_{4} /$ flat (b) type layers. The building fragments ("bricks") of the layers are also shown in the figure.

nected to $42 / 3$ octahedra via edges and to $2^{2} / 3$ others via corners. In lepidocrocite, on average, each $\mathrm{Ti}-\mathrm{O}_{6}$ octahedron is connected to 6 octahedra via edges and to 2 others via corners. Thus the number of corner sharing octahedra in trititanate $\mathrm{H}_{2} \mathrm{Ti}_{3} \mathrm{O}_{7}$ is higher than that in lepidocrocite $\mathrm{H}_{x} \mathrm{Ti}_{2-x / 4} \square_{x / 4} \mathrm{O}_{4}$. Titanium atoms from adjacent, edgesharing octahedral units are separated at distances of $3.1 \AA$ and those from adjacent, corner-sharing octahedra - at $3.7 \AA$ (marked with a blue diamond on Fig. 5). As the data presented in Fig. 5 show the peak at $3.7 \AA$ in the experimental $\mathrm{PDF}$ is better reproduced by the $\mathrm{H}_{2} \mathrm{Ti}_{3} \mathrm{O}_{7^{-}}$ type model. This finding supports the model of Chen et al. [4] featuring the titanate nanotube walls as a scroll of corrugated layers of $\mathrm{Ti}-\mathrm{O}_{6}$ octahedra. Refined model parameters are summarized in Table 1 . They are of the type yielded by traditional crystallography and thus may be used conveniently to compute and understand various structure-dependent properties of titanate nanotubes. We used them to build real size models of titanate nanotubes. Such models may be very helpfull in examining and understanding the observed strong relationship between the band gap and diameter of titanate nanotubes. The models were built through subsequent linear translations and rotations of the basic building units of trititanate and lepidocrocite, fragmented and flat, layers, respectivelly. The layers were kept as rigid as they occur in the corresponding crystals, i.e. the cylindrical shape of titanate nanotubes was achieved only by rotating and linearly translating adjacent layer sections without breaking or generating atomic bonds. As built, the model nanotubes are approx. $9 \mathrm{~nm}$ in diameter and, for nanotubes of length $\sim 5 \mathrm{~nm}$ were comprised of $\sim 30,000$ atoms. Model nanotubes are shown in Fig. 6 and the corresponding atomic PDFs - in Fig. 7. As can be seen in the figure the atomic PDFs for both nanotube models approximate the experimental data reasonably well with that based on corrugated layers again performing somewhat better, showing that the walls of titanate nanotubes may well be viewed as scrolls of layers shown in Fig. 4a. A notable difference between the calculated PDFs for a scroll of corrugated layers (Fig. 6a) and a stack of straight corrugates layers (Fig. 4b) is seen in the vicinity of the $3.7 \AA$ peak [26]. The nanotube model (bent layers) does not fit this peak as well as a crystal-type model (straight layers) does (compare the model results shown in Fig. 5 and 7). This can be explained by the fact that in the model constructed by us the bending of layers was achieved by rotating and translating linearly identical

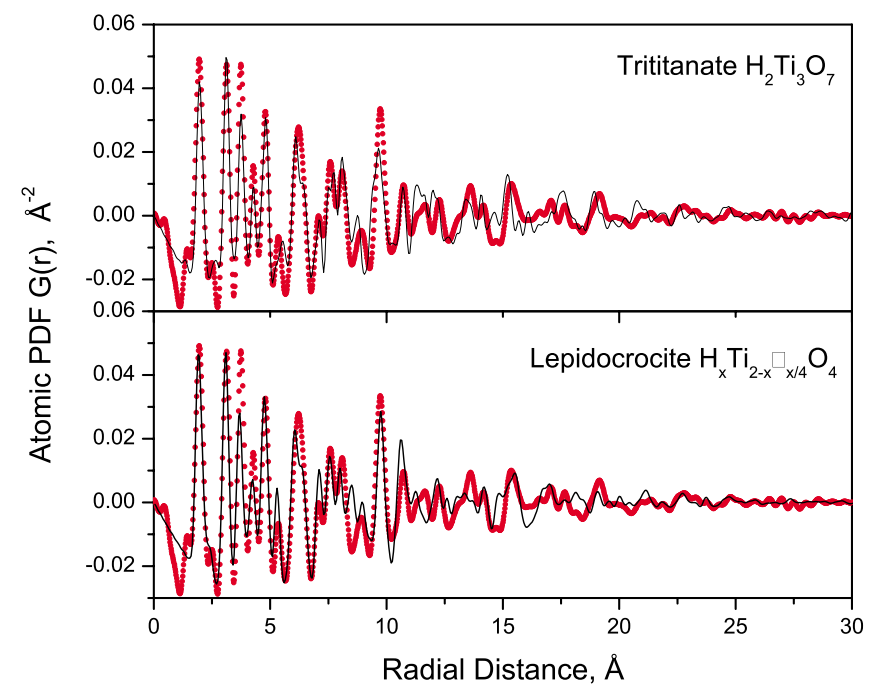

Fig. 7. Comparison between the experimental PDF for titania nanotubes (symbols in red) and calculated PDFs (solid lines in black) for the model nanotubes shown in Fig. 6.

layer fragments with respect to each other which, obviously, introduced a unrealistically wide distribution of distances between $\mathrm{Ti}$ atoms from adjacent corner-sharing octahedra. Near neighbour Ti-Ti distances in the nanotube walls exhibit a narrower $\mathrm{Ti}-\mathrm{Ti}$ distance distribution as the experimental data show. A more realistic model of titania nanotubes should reproduce this distribution better. To achieve this it may be necessary to introduce some flexibility in (i.e. reduce the rigidity of) the layer building units as well as involve shear and not only linear translations. This may help better take into account the fact that the corrugated layers of $\mathrm{Ti}-\mathrm{O}_{6}$ octahedra building the walls of real titanate nanotubes are neither perfect nor stacked in registry as they are in crystalline trititanate.

Table 1. Fractional coordinates of atoms inside the trititanate-type unit cell/repetitive unit used to describe the structure of titania nanotubes as refined against the experimental PDF data. All atoms are in position $4 i(x, 0, z)$ of the space group $C 2 / m$ and the lattice parameters are $a^{1}=35.466 \AA, b=3.7568 \AA, c=9.759 \AA, \beta=101.46^{\circ}$.

\begin{tabular}{lll}
\hline Atom & $x$ & $z$ \\
\hline Ti1 & 0.2339 & 0.2198 \\
Ti2 & 0.2183 & 0.5438 \\
Ti3 & 0.1989 & 0.8467 \\
O1 & 0.2094 & 0.0430 \\
O2 & 0.1949 & 0.3257 \\
O3 & 0.1730 & 0.6256 \\
O4 & 0.1337 & 0.8696 \\
O5 & 0.2858 & 0.1289 \\
O6 & 0.2602 & 0.4164 \\
O7 & 0.2412 & 0.7440
\end{tabular}

1 The edge of the unit cell/ repetitive unit " $a$ " is chosen long enough to eliminate distances (PDF peaks) coming from two adjacent layers. Note this " $a$ " is not the distance between the individual shells/layers but a parameter used to describe the repetitive pattern/ periodic structure of nanotube walls. 


\section{Conclusions}

High-energy X-ray diffraction coupled to atomic PDF data analysis can reveal the atomic ordering in nanosized materials in very good detail, even if they have the complex morphology of nanotubes. The approach has the potential to become a routine experimental tool given the rapid increase in the number of synchrotron sources worldwide.

Acknowledgments. The work was supported by the NSF through Grant DMR 0304391(NIRT) and CMU through Grant REF C602281. Use of the Advanced Photon Source was supported by the U.S. Department of Energy, Office of Basic Energy Sciences, under Contract DE-AC02-06CH11357.

\section{References}

[1] Kasuga, T.; Hiramatsu, M.; Hoson. A.; Sekino, T.; Niihara, K.: Formation of titanium oxide nanotube. Langmuir 14 (1998) 3160-3163.

[2] Kasuga, T.; Hiramatsu, M.; Hoson, A.; Sekino, T.; Niihara, K.: Titania nanotubes prepared by chemical processing. Adv. Mater. 11 (1999) 1307-1311.

[3] Bavykin D. V.; Friedrich J. M.; Walsh, F. C.: Protonated titanates and $\mathrm{TiO}_{2}$ nanostructured materials: synthesis, properties and applications. Adv. Mater. 18 (2006) 2807-2824.

[4] Chen, Q.; Peng, L.-M.: Structure and applications of titanate and related nanostructures. Int. J. Nanotechnology 4 (2007) 4465 .

[5] Chen, Q.; Du, G. H.; Zhang, S.; Peng, L.-M.: The structure of trititanate nanotubes. Acta Cryst. B58 (2002) 587-593.

[6] Zhang, S.; Chen, Q.; Peng, L.-M.: Structure and formation of $\mathrm{H}_{2} \mathrm{Ti}_{3} \mathrm{O}_{7}$ nanotubes in alkali environment. Phys. Rev. B71 (2005) 014104.

[7] Zhang, S.; Peng, L.-M.; Chen, Q.; Du, G. H.; Dawson, G.; Zhou, W. Z.: Formation Mechanism of $\mathrm{H}_{2} \mathrm{Ti}_{3} \mathrm{O}_{7}$ nanotubes. Phys. Rev. Lett. 91 (2003) 256103.

[8] Enyashin, A. N.; Seifert, G.: Structure, stability and electronic properties of $\mathrm{TiO}_{2}$ nanostructures. phys. stat. sol. (b) 242 (2005) $1361-1370$.

[9] Ma, R.; Bando, Y.; Sasaki, T.: Nanotubes of lepidocrocite titanates. Chem. Phys. Lett. 380 (2003) 577-582.

[10] Nakahira, A.; Kato, W.; Tamai, M.; Isshiki, T.; Nishio, K.: Synthesis of nanotube from a layered $\mathrm{H}_{2} \mathrm{Ti}_{4} \mathrm{O}_{9} \mathrm{H}_{2} \mathrm{O}$ in a hydrothermal treatment using various titania sources. J. Mater. Sci. 39 (2004) 4239-4245.

[11] Morgado, E.; de Abreu, M. A. S.; Pravia, O. R. C.; Marinkovic, B. A.; Jardim, P. M.; Rizzo, F. C.; Araujo, A. S.: A study on the structure and thermal stability of titanate nanotubes as a function of sodium content. Solid State Sci. 8 (2006) 888-900.

[12] Ferreira, O. P.; Filho, A. G. S.; Filho, J. M.; Alves, O. L.: Unveiling the structure and composition of titanium oxide nanotubes through ion exchange chemical reactions and thermal decomposition processes. J. Braz. Chem. Soc. 17 (2006) 393-402.

[13] Ma, R.; Sasaki, T.; Bando, Y.: Layer-by-layer assembled multilayer films of titanate nanotubes, Ag- or Au-loaded nanotubes, and nanotubes/nanosheets with polycations. J. Am. Chem. Soc. 126 (2004) 10382-10388.

[14] Ma, R.; Sasaki, T.; Bando, Y.: Alkali metal cation intercalation properties of titanate nanotubes. Chem. Commun. (2005) 948950.

[15] Suzuki, Y.; Yoshikawa, S.: Synthesis and thermal analyses of $\mathrm{TiO}_{2}$-derived nanotubes prepared by the hydrothermal method. J. Mater. Res. 19 (2004) 982-985.

[16] Petkov, V.; Ohta, T.; Hou, Y.; Ren. Y.: Atomic-scale structure of nanocrystals by high-energy $\mathrm{X}$-ray diffraction and atomic pair distribution function analysis: study of $\mathrm{Fe}_{x} \mathrm{Pd}_{100-x}(x=0,26$, 28, 48) nanoparticles. J. Phys. Chem. C 111 (2007) 714-720.

[17] Petkov, V.; Gateshki, M.; Niederberger, M.; Ren. Y.: Atomicscale structure of nanocrystalline $\mathrm{Ba}_{x} \mathrm{Sr}_{1-x} \mathrm{TiO}_{3}(x=1,0.5,0)$ by X-ray diffraction and the atomic pair distribution function technique. Chem. Mater. 18 (2006) 814-821.

[18] Gateshki, M.; Hwang, S.-J.; Park, D. H.; Ren. Y.; Petkov, V.: Structure of exfoliated titanate nanosheets determined by atomic pair distribution function analysis. Chem. Mater. 16 (2004) 5153-5157.

[19] Gateshki, M.; Yin, S.; Ren, Y.; Petkov, V.: Titania Polymorphs by soft chemistry: Is there a common structural pattern? Chem. Mater. 19 (2007) 2512-2518.

[20] Egami, T.; Billinge, S. J. L.: Underneath the Bragg Peaks. Structural Analysis of Complex Materials; Pergamon: Oxford, U.K., 2003.

[21] Wagemaker, M.; Kearley, G. J.; van Well, A. A.; Mutka, H.; Mulder, F. M.: Multiple Li positions inside oxygen octahedra in lithiated $\mathrm{TiO}_{2}$ anatase. J. Am. Chem. Soc. 125 (2003) 840-848.

[22] Klug, H. P.; Alexander, L. E.: X-ray Diffraction Procedures for Polycrystalline Materials; Wiley: New York, 1974.

[23] Petkov, V.: RAD, a program for analysis of X-ray diffraction data from amorphous materials for personal computers. J. Appl. Crystallogr. 22 (1989) 387

[24] Petkov, V.; Zavalij, P. Y.; Lutta, S.; Whittingham, M. S.; Parvanov, V.; Shastri, S.: Structure beyond Bragg: study of $\mathrm{V}_{2} \mathrm{O}_{5}$ nanotubes. Phys. Rev. B69 (2004) 085410.

[25] Proffen, Th.; Billinge, S. J. L.: PDFFIT, a program for full profile structural refinement of the atomic pair distribution function. J. Appl. Crystallogr. 32 (1999) 572-575.

[26] The building blocks of the lepidocrocite model are smaller than those of the trititanate one and, hence, require less rotation/translation when used to build a nanotube wall. In this way, $\mathrm{Ti}-\mathrm{Ti}$ distances between adjacent octahedra remain close to those in flat layers as seen in the hardly changing 3.1 and $3.7 \AA$ PDF peak of the lepidocrocite model. 